\title{
A cross-sectional study of the association between mobile phone use and symptoms of ill health
}

\author{
Yong Min Cho ${ }^{1}$, Hee Jin $\mathrm{Lim}^{2}$, Hoon Jang ${ }^{2}$, Kyunghee $\mathrm{Kim}^{2}$, Jae Wook Choi ${ }^{2}$, Chol Shin ${ }^{3}$, \\ Seung Ku Lee ${ }^{3}$, Jong Hwa Kwon ${ }^{4}$, Nam Kim ${ }^{5}$ \\ ${ }^{1}$ Institute for Life and Environment Technology, Smartive Corporation, Seoul; ${ }^{2}$ Institute for Occupational and Environmental \\ Health, Korea University, Seoul; Institute of Human Genome Study, Korea University Ansan Hospital, Ansan; ${ }^{4} E M$ \\ Environment Research Team, Electronics and Telecommunications Research Institute, Daejeon; ${ }^{5}$ Division of Information and \\ Communication Engineering, Chungbuk National University, Chungju, Korea
}

Objectives This study analyzed the associations between mobile phone call frequency and duration with non-specific symptoms.

Methods This study was conducted with a population group including 532 non-patient adults established by the Korean Genome and Epidemiology Study. The pattern of phone call using a mobile phone was investigated through face-to-face interview. Structured methods applied to quantitatively assess health effects are Headache Impact Test-6 (HIT6), Psychosocial Well-being Index-Short Form, Beck Depression Inventory, Korean-Instrumental Activities of Daily Living, Perceived Stress Scale (PSS), Pittsburgh Sleep Quality Index, and 12-item Short Form Health Survey where a higher score represents a higher greater health effect.

Results The average daily phone call frequency showed a significant correlation with the PSS score in female subjects. Increases in the average duration of one phone call were significantly correlated with increases in the severity of headaches in both sexes. The mean (standard deviation) HIT-6 score in the subgroup of subjects whose average duration of one phone call was five minutes or longer was 45.98 (8.15), as compared with 42.48 (7.20) in those whose average duration of one phone call was $<5$ minutes. The severity of headaches was divided into three levels according to the HIT-6 score (little or no impact/moderate impact/substantial or severe impact), and a logistic regression analysis was performed to investigate the association between an increased phone call duration and the headache severity. When the average duration of one phone call was five minutes or longer, the odds ratio (ORs) and the 95\% confidence intervals (Cls) for the moderate impact group were 2.22 and 1.18 to 4.19 , respectively. The OR and $95 \% \mathrm{Cl}$ for the substantial or severe impact group were 4.44 and 2.11 to 8.90 , respectively.

Conclusions Mobile phone call duration was not significantly associated with stress, sleep, cognitive function, or depression, but was associated with the severity of headaches.

Keywords Mobile phones, Headaches, Headache Impact Test-6, Non-specific symptoms

\author{
Correspondence: Jae Wook Choi \\ 73 Inchon-ro, Seongbuk-gu, Seoul 02841, \\ Korea \\ Tel: +82-2-2286-1470 \\ Fax: $+82-2-927-7220$ \\ E-mail: shine@korea.ac.kr
}

Received: September 5, 2016

Accepted: October 21, 2016

Published: October 26, 2016

This article is available from: $h$ ttp://e-eht.org/

\section{Introduction}

With the development of telecommunication technology, mobile phones have been rapidly introduced to the public over the past few decades. The number of telecommunication subscrib- ers in South Korea (hereafter Korea) in 2009 was greater than the entire population [1], which is also true worldwide [2]. Communication using a mobile phone has become a part of daily living of contemporaries.

A mobile phone terminal, which is a type of low-power, wire- 
less transmitter-receiver, is a source of radiofrequency-electromagnetic fields (RF-EMF) [3]. In addition, mobile phone calls require the establishment of mobile phone base stations. Therefore, the use of mobile phones increases the risk of RF-EMF exposure to the general population [4].

The most important health effects of RF-EMF exposure due to mobile phone use are brain tumors. Previous large-scale, epidemiological studies reported that mobile phone use might increase the risk of brain tumors, including glioma, meningioma, and acoustic neuroma $[5,6]$. On the basis of these studies, the International Agency for Research on Cancer classified the RFEMF from mobile phones as possibly carcinogenic to humans (Group 2B) $[7,8]$. However, a correlation between exposure to RF-EMF and health effects does not have a solid scientific basis [9], which makes the issue controversial. Nevertheless, on the basis of precautionary principle, efforts should be made to identify the health effects of RF-EMF exposure.

Few studies have clearly explained the cause and effect relationships between various types of health effects and mobile phone use. Some studies have shown that mobile phone use may cause non-specific symptoms, such as fatigue, headaches, dizziness, skin symptoms, a burning sensation, eye pain, discomfort, sleep disorders, and anxiety [10]. Non-specific symptoms, which may be defined as "medically unexplained symptoms," may occur in a population even when the level of environmental risk factors is lower than required by the safety standards [11].

Previous studies have suggested that headaches, which are a type of non-specific symptom, might be correlated with mobile phone use [12-14]. This cross-sectional study analyzed the correlations between the characteristics of mobile phone usage (frequency and duration) and symptoms related to daily living, such as headaches, stress, cognitive function, depression, and sleep patterns.

\section{Materials and Methods}

\section{Study Subjects}

The present study was conducted with a non-patient popula- tion group established by the Korean Genome and Epidemiology Study (KoGES-Ansan). The KoGES-Ansan is a communitybased prospective cohort study, and which has been conducted since 2011 [15]. The subjects of the present cross-sectional study are participants of the KoGES-Ansan who visited the Korea University Ansan Hospital between 2012 and 2013 for a medical examination. All subjects consented to participation in the present study. In the KoGES-Ansan, a regular medical examination is performed at 2-year intervals when the subjects visit the hospital. Among the subjects who underwent the medical examination in 2012 or 2013, 532 subjects underwent a follow-up examination two years later in 2014 or 2015 . The results of the investigation performed with the 532 subjects in 2012 and 2013 are provided on this article, whereas the follow-up data, including the results of the investigation performed in 2014 and 2015, will be provided in our future work.

All subjects were adults over the age of 40 who had used a mobile phone for several years. The investigation items of the present study were approved by the Centers for Disease Control and Prevention (Korea CDC) and the human subjects review committee in Korea University supervising the KoGES-Ansan. In addition, informed consent was obtained from all subjects.

\section{Data Collection and Variables}

Professional medical staff performed the survey and examination. The survey of each item was conducted via a one-to-one interview. The survey items associated with the characteristics of mobile phone use included the average number of daily phone calls using a mobile phone. In addition, the average duration of one phone call in minutes was investigated. Mobile phone use included both transmission and reception, but excluded text message transmission and reception, and the use of the Internet and other information services. To reduce recall bias, mobile phone use was assessed as a breakdown of the mobile phone bill provided in a hard copy or through a mobile phone application.

Table 1 summarizes the survey items that were investigated to

Table 1. Heath effect survey items

\begin{tabular}{llclc}
\hline Health outcome & Applied tool & Score range & & Meaning \\
\hline Headache & HIT-6 & $36-78$ & A higher score indicates a more severe headache & Reference \\
Psychosocial well-being & PWI-SF & $0-54$ & A higher score indicates a higher risk of stress & 16,17 \\
Depression & BDI & $0-63$ & A higher score indicates more severe depression & 18,19 \\
Dementia (daily activities) & K-IADL & $7-21$ & A higher score indicates a higher dependency & 19,20 \\
Stress & PSS & $0-40$ & A higher score indicates a higher level of recognized stress & 21 \\
Sleep quality & PSQI & $0-21$ & A higher score indicates poorer sleep quality & 22,23 \\
Physical and mental health & SF-12 & $12-56$ & A higher score indicates a lower health level & 24,25 \\
\end{tabular}

HIT-6, Headache Impact Test-6; PWI-SF, Psychosocial Well-being Index-Short Form; BDI, Beck Depression Inventory; K-IADL, Korean-Instrumental Activities of Daily Living; PSS, Perceived Stress Scale; PSQI, Pittsburgh Sleep Quality Index; SF-12, 12-item Short Form Health Survey. 
understand the health effects of mobile phone use on the subjects. First, the Headache Impact Test-6 (HIT-6), which is a structured survey inventory used to assess the adverse effects of headaches on quality of life, was employed to assess the correlation between mobile phone use and headaches $[16,17]$. The Psychosocial Well-being Index-Short Form (PWI-SF) was utilized to assess the state of mental health $[18,19]$, and the Beck Depression Inventory (BDI) was employed to assess the level of depression in the subjects $[19,20]$. The Korean-Instrumental Activities of Daily Living (K-IADL) was used as an inventory to assess the occurrence of dementia due to cognitive dysfunction in the elderly [21]. We also used the Perceived Stress Scale (PSS) for the assessment of recognized stress [22,23], the Pittsburgh Sleep Quality Index (PSQI) for the assessment of sleep quality $[24,25]$, and the 12-item Short Form Health Survey (SF-12) for the assessment of the overall health level $[26,27]$. The majority of these survey inventories were developed in other countries, and the reliability and validity of these inventories have been recognized. The Korean versions of the inventories have also been recognized in terms of their reliability and validity.

With regard to headaches, the severity was divided into three groups according to the HIT- 6 score for further evaluation. The HIT-6 score ranges from 36 to 78 points: a score less than or equal to 49 points was expressed as "little or no impact of headache"; a score between 50 and 55 as "moderate impact of headache"; and a score greater than 55 as "substantial or severe impact of headache" [28].

The average duration of one phone call in minutes was divided into long-time use and short-time use. The reference value of long-time use according to the specific absorption rate, which represents the thermal effect of electromagnetic radiation, is six minutes [29]. The cut-off for long-time use of mobile phone call was established as five minutes per call in the recent epidemiological study [30]. In this study, five minutes of call duration per call was approximately 80th percentile. In addition, in the several epidemiological studies have already reported relationships between the call duration and health outcomes, cut-off lines of "heavy use" for call duration were defined the 3rd quartile or 80th to 90th of all data $[31,32]$. Therefore, an average duration of one phone call equal to or longer than five minutes was expressed as a long-time use, while an average duration of one phone call shorter than five minutes was expressed as a short-time use.

\section{Statistical Analysis}

In the present study, the intensity of mobile phone call use was estimated using the variables of the daily average number of calls using a mobile phone and the average duration of one phone call. We analyzed the correlations between these variables and the scores of various assessments of health status by performing a linear regression analysis. In addition, Student's $t$-tests were performed to compare the severity of headaches between the long-time and short-time use subjects. Odds ratios (ORs) and 95\% confidence intervals (CIs) were calculated using a logistic regression analysis to assess the significance of differences in the HIT-6 scores between the little or no, moderate, or substantial or severe impact subgroups in subjects whose average mobile phone call duration was equal to or longer than five minutes.

The SPSS version 23.0 (IBM Corp., Armonk, NY, USA) was used to perform statistical analyses. Significance was tested at $95 \%$ and $99 \%$ statistical credibility ( $p<0.05$ and 0.01 ).

\section{Results}

Table 2 shows the demographic characteristics of the subjects. A total of 532 subjects visited the hospital between 2012 and 2013 to undergo the survey and a health screening. The average age of the subjects was 57 years. The median daily average number of phone calls was five. The median of the average duration of one phone call was 1.5 minutes. The duration of one phone call was less than five minutes in approximately $80 \%$ of the subjects. The significant differences of mobile phone use characteristics and the distribution of HIT-6 score between both sexes were not found (data were not shown).

Correlations between each variable for mobile phone uses and health outcomes are shown on Table 3. The variable of average frequency of call per day was significantly correlated with PSS (both sexes) and HIT-6 (only for males) as well as call duration with HIT-6 (both sexes).

The results of the linear regression analysis of the mobile phone call characteristics and health effect variables showed a significant positive correlation between the average duration of one phone call and the severity of headaches in both sexes (Ta-

Table 2. Demographic characteristics of the subjects

\begin{tabular}{lcc}
\hline Variable & $\mathrm{n}(\%)$ & $\begin{array}{c}\text { Median } \\
\text { (1st-3rd quartile) }\end{array}$ \\
\hline $\begin{array}{l}\text { Sex } \\
\quad \text { Female }\end{array}$ & $298(56.0)$ & \\
$\quad$ Male & $234(44.0)$ & \\
Age & & $57.0(53.0,63.0)$ \\
Average frequency of calls (n/d) & & $5.0(3.0,10.0)$ \\
$\quad<5$ & $214(40.2)$ & \\
$5-<10$ & $156(29.3)$ & \\
& $143(26.9)$ & \\
Average call duration (min/call) & & \\
$\quad<5$ & $426(80.1)$ & \\
$5-<10$ & $35(6.6)$ & \\
$\geq 10$ & $47(8.8)$ & \\
\hline
\end{tabular}


Table 3. Pearson's correlation coefficient between each data investigated

\begin{tabular}{|c|c|c|c|c|c|c|c|c|}
\hline & Frequency & Call duration & HIT-6 & BDI & K-IADL & PSS & PSQI & SF-12 \\
\hline \multicolumn{9}{|l|}{ Female } \\
\hline Frequency & 1 & & & & & & & \\
\hline Call duration & 0.013 & 1 & & & & & & \\
\hline HIT-6 & -0.011 & $0.191^{\star *}$ & 1 & & & & & \\
\hline $\mathrm{BDI}$ & 0.049 & 0.059 & $0.148^{*}$ & 1 & & & & \\
\hline K-IADL & 0.026 & -0.052 & 0.069 & $0.182^{\star *}$ & 1 & & & \\
\hline PSS & $0.128^{*}$ & 0.008 & $0.159^{*}$ & $0.231^{* *}$ & 0.059 & 1 & & \\
\hline PSQI & -0.029 & -0.001 & $0.205^{\star \star}$ & $0.295^{\star *}$ & $0.116^{*}$ & 0.017 & 1 & \\
\hline SF-12 & -0.030 & -0.014 & $0.153^{\star *}$ & $0.464^{\star \star}$ & $0.265^{\star \star}$ & 0.112 & $0.381^{\star \star}$ & 1 \\
\hline \multicolumn{9}{|l|}{ Male } \\
\hline Frequency & 1 & & & & & & & \\
\hline Call duration & 0.007 & 1 & & & & & & \\
\hline HIT-6 & $0.241^{\star *}$ & $0.227^{\star \star}$ & 1 & & & & & \\
\hline BDI & -0.052 & -0.031 & $0.175^{\star \star}$ & 1 & & & & \\
\hline K-IADL & -0.011 & -0.035 & -0.041 & $0.240^{\star \star}$ & 1 & & & \\
\hline PSS & $0.195^{\star}$ & 0.012 & $0.247^{\star \star}$ & 0.006 & -0.041 & 1 & & \\
\hline PSQI & -0.088 & -0.077 & 0.028 & $0.387^{\star \star}$ & 0.045 & 0.028 & 1 & \\
\hline SF-12 & -0.044 & -0.085 & -0.063 & $0.502^{\star \star}$ & $0.280^{\star \star}$ & -0.075 & $0.220^{\star \star}$ & 1 \\
\hline
\end{tabular}

Frequency, average frequency of call per day; Call duration, average call duration per call (min/call); HIT-6, Headache Impact Test-6; BDI, Beck Depression Inventory; K-IADL, Korean-Instrumental Activities of Daily Living; PSS, Perceived Stress Scale; PSQl, Pittsburgh Sleep Quality Index; SF-12, 12-item Short Form Health Survey. ${ }^{*} p<0.05,{ }^{* *} p<0.01$.

Table 4. Results of a linear regression analysis with respect to mobile phone call characteristics and health effect variables

\begin{tabular}{|c|c|c|c|c|c|c|c|}
\hline & HIT-6 & PWI-SF & $\mathrm{BDI}$ & K-IADL & PSS & PSQI & SF-12 \\
\hline \multicolumn{8}{|l|}{ Female $(n=298)$} \\
\hline Frequency & $\begin{array}{c}0.02 \\
(-0.12,0.16)\end{array}$ & $\begin{array}{c}0.42 \\
(-0.14,0.97)\end{array}$ & $\begin{array}{c}0.01 \\
(-0.12,0.14)\end{array}$ & $\begin{array}{c}0.02 \\
(-0.01,0.05)\end{array}$ & $\begin{array}{c}0.11 \\
(0.02,0.20)^{*}\end{array}$ & $\begin{array}{c}-0.02 \\
(-0.07,0.04)\end{array}$ & $\begin{array}{c}-0.05 \\
(-0.16,0.07)\end{array}$ \\
\hline Call time (min) & $\begin{array}{c}0.07 \\
(0.02,0.13)^{*}\end{array}$ & $\begin{array}{c}-0.01 \\
(-0.07,0.05)\end{array}$ & $\begin{array}{c}<-0.01 \\
(-0.05,0.05)\end{array}$ & $\begin{array}{c}<-0.01 \\
(-0.02,0.01)\end{array}$ & $\begin{array}{c}<0.01 \\
(-0.11,0.12)\end{array}$ & $\begin{array}{c}<0.01 \\
(-0.02,0.03)\end{array}$ & $\begin{array}{c}-0.01 \\
(-0.06,0.04)\end{array}$ \\
\hline \multicolumn{8}{|l|}{ Male $(n=234)$} \\
\hline Frequency & $\begin{array}{c}0.04 \\
(-0.01,0.10)\end{array}$ & $\begin{array}{c}-0.08 \\
(-0.20,0.04)\end{array}$ & $\begin{array}{c}-0.03 \\
(-0.07,0.01)\end{array}$ & $\begin{array}{c}<0.01 \\
(-0.01,0.01)\end{array}$ & $\begin{array}{c}0.02 \\
(-0.03,0.07)\end{array}$ & $\begin{array}{c}-0.01 \\
(-0.03,0.01)\end{array}$ & $\begin{array}{c}-0.03 \\
(-0.06,0.01)\end{array}$ \\
\hline Call time (min) & $\begin{array}{c}0.10 \\
(0.04,0.17)^{\star \star}\end{array}$ & $\begin{array}{c}-0.07 \\
(-0.15,0.02)\end{array}$ & $\begin{array}{c}0.04 \\
(-0.01,0.09)\end{array}$ & $\begin{array}{c}<-0.01 \\
(-0.01,0.01)\end{array}$ & $\begin{array}{c}0.05 \\
(-0.03,0.12)\end{array}$ & $\begin{array}{c}<0.01 \\
(-0.02,0.03)\end{array}$ & $\begin{array}{c}-0.01 \\
(-0.05,0.03)\end{array}$ \\
\hline
\end{tabular}

The numbers in parenthesis mean the regression coefficient (95\% confidence interval).

Adjusted variables were age, alcohol consumption, smoking, and body mass index.

HIT-6, Headache Impact Test-6; PWI-SF, Psychosocial Well-Being Index-Short Form; BDI, Beck Depression Inventory; K-IADL, Korean-Instrumental Activities of Daily Living; PSS, Perceived Stress Scale; PSQI, Pittsburgh Sleep Quality Index; SF-12, 12-item Short Form Health Survey.

${ }^{*} p<0.05,{ }^{* *} p<0.01$.

ble 4). The daily average number of phone calls was significantly correlated with a higher PSS score in female, but not male, subjects. The PWI-SF stress index, the BDI, the cognitive dysfunction (dementia) index (K-IADL), and the PSQI scores, as well as the overall health status index (SF-12) scores, were not significantly correlated with the daily average number of mobile phone calls and the duration of one phone call. Only headache severity (HIT-6 score) showed a significant correlation with the phone call characteristics.

Figure 1 shows the HIT-6 score distribution in the long-time and short-time use groups. The mean (standard deviation) HIT-6 score was 42.48 (7.20) in the short-time use group $(\mathrm{n}=417)$, and $45.98(8.15)$ in the long-time use group $(n=80)$. These results indicated that the mean HIT-6 score was significantly higher in the long-time use group compared with the short-time use group ( $p<0.001$ by Student's $t$-test).

The severity of headaches was divided into three levels according to the HIT-6 score, and a logistic regression analysis was performed to assess the severity of headaches in the long-time use group (Figure 2). Compared with the "little or no impact of headache" subgroup, which is the weakest headache severity, the OR (95\% CI) of subjects in the long-time use group who were classified as having a "moderate impact of headache" was 2.22 (1.78 to 4.19), and that classified as having a "substantial or 


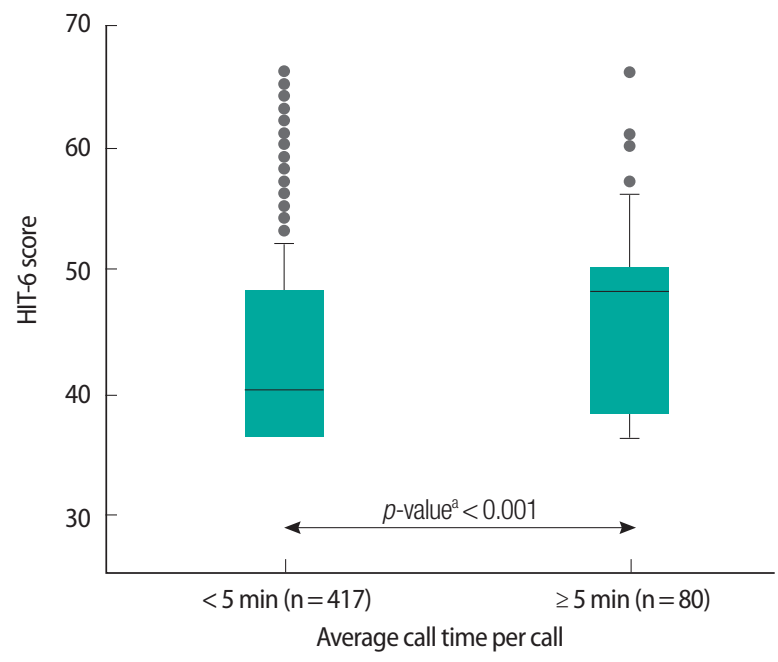

Figure 1. Comparison of Headache Impact Test-6 (HIT-6) score distribution between the group of subjects whose average duration of one phone call was 5 minutes or longer and the group of subjects whose average du-

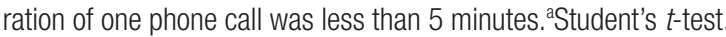

severe impact of headache" was 4.44 (2.11 to 8.90$)$. The Pearson's correlation coefficient with the HIT-6 score calculated with the individual phone call duration as a continuous variable was 0.221 , which was also significant $(p<0.001)$.

\section{Discussion}

This study showed that there was a significant positive correlation between the average duration of one phone call and the severity of headaches in both sexes. Conversely, the daily average number of phone calls showed no correlation with the health effect items investigated in the present study, and the duration of one phone call was not associated with health effects other than headache severity independently, although headaches had significant correlated with depression, stress, sleep and health status.

In a large population-based, cross-sectional study conducted in 2009, Berg-Beckhoff et al. [33] employed inventories similar to those used in the present study to assess the correlation of farfield (from a mobile phone base station) exposure to RF-EMF with sleep quality (PSQI), headache severity (HIT-6), psychosomatic complaints (von Zerssen list), physical and mental health (SF-36), and chronic stress (Trier Inventory for Chronic Stress), and reported that the above-mentioned health effects were not correlated with the RF-EMF exposure levels.

However, mobile phone calls are associated with near-field exposure to RF-EMF. The correlation between mobile phone use and headaches has previously been reported in several studies. Similar to the result of the present study, it has been reported that the headache severity perceived by mobile phone users was

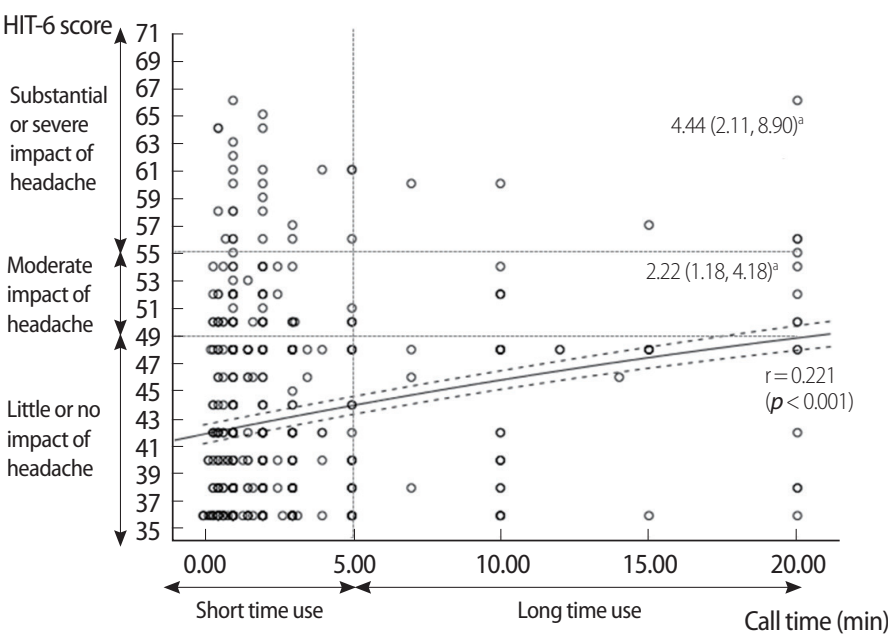

Figure 2. Correlation between the average duration of one phone call and the severity of headaches, with reference to 5 -minute average duration of one phone call. HIT-6, Headache Impact Test-6; r, Pearson's correlation

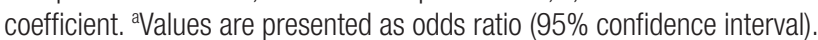

greater as the duration of a phone call using a mobile phone was increased $[12,34,35]$. In addition, it has been reported that the heat sensation adjacent to the ears was increased as the duration of a phone call using a mobile phone was increased [36,37], and a considerable number of the subjects who complained of this heat sensation also complained of headaches [38]. If a headache is defined as all types of pain experienced at the head, the heat sensation at the head, including the ears, may be one of the causes of headaches. The mechanism by which heat sensations might cause headaches has not clearly been identified, and the effect of heat sensations on other types of health effects, including mental health symptoms, a decreased quality of sleep, and the overall health status, as well as the severity of headaches, has not been sufficiently described. However, the results of the present study may be reasonably explained if a long-time phone call using a mobile phone causes heat sensations at the part of the body in contact with the mobile phone, and the heat sensation leads to a headache.

In the present study, the mobile phone use characteristics were investigated through a survey. Since the RF-EMF exposure due to mobile phone calls was not actually measured, the results might not have meaning as a quantitative exposure assessment. Therefore, the limitation of the present study was the lack of evidence that the headache effect identified in the subjects was caused by RF-EMF exposure. In addition, since the present study was based on a cross-sectional design, the results might not clearly explain the cause and effect relationship between mobile phone calls and health effects. This is also a limitation of studies applied epidemiologic designs. Experimental trials with quantitative measuring of RF-EMF exposure levels have diffi- 
culties to be applied to large-size population and to find representative value of exposure. The situation of mobile phone use may mean behaviors of study subjects for a long time rather than temporary exposure levels. There are many epidemiological studies reported relationship between mobile phone and health effects through the methods of questionnaires like our study [10]. Some researchers questioned the information collected through interviews or questionnaires from the point of view of reliability of exposure assessment. To reduce recall biases, the way including reviewing mobile phone subscriber records as well as self-reports or interviews are recommended [39] like our study.

Despite a number of limitations, the present study demonstrated that mobile phone call duration was positively correlated with headache severity. The correlation between mobile phone call duration and the headache effect suggests that the duration of mobile phone calls might be correlated with other electromagnetic hypersensitivity symptoms (EHS), with the exception of stress, sleep, and physical and mental health, in a different manner.

This study aimed to quantitatively assess the effects of mobile phone use characteristics on various types of health effects. However, the health effect symptoms, with the exception of headache severity, showed no significant correlation with mobile phone use. The symptoms investigated in the present study, which included a decreased quality of sleep, stress, and the overall health status of the subjects, as well as headaches, were representative EHS. These symptoms are considered as non-specific and subjective, and thus the medical probability of these symptoms is unclear. Nevertheless, it is not appropriate to place all symptoms into one category of 'non-specific' symptoms. It is necessary to recognize that the various types of symptoms that might be associated with mobile phone use might have different patterns and underlying mechanisms, and thus more extensive investigations of the symptoms are required.

This cross-sectional study is part 1 of our whole study including cross-sectional and follow-up design with a duration of two years. In the following study, results of follow-up will be suggested as an article of part 2.

\section{Acknowledgements}

This work was supported by grants from the IT R\&D program of MSIP/IITP (B0138-15-1002, Study on the EMF exposure control in smart society) and the Korea Centers for Disease Control and Prevention (2012-E71005-00 and 2013-E71005-00).

\section{Conflict of Interest}

The authors have no conflicts of interest associated with the material presented in this paper.

\section{ORCID}

Yong Min Cho http://orcid.org/0000-0002-8999-8916

Hee Jin Lim http://orcid.org/0000-0001-5295-0137

Hoon Jang http://orcid.org/0000-0001-8549-8001

Kyunghee Kim http://orcid.org/0000-0002-5333-9716

Jae Wook Choi http://orcid.org/0000-0002-1996-7524

Chol Shin http://orcid.org/0000-0002-2928-8576

Seung Ku Lee http://orcid.org/0000-0001-5120-5557

Jong Hwa Kwon http://orcid.org/0000-0002-2132-7403

Nam Kim http://orcid.org/0000-0001-8109-2055

\section{References}

1. Korea Communications Commission. The status of fixed and mobile telecommunication service subscribers [cited 2016 Jun 27]. Available from: http://www.kcc.go.kr/user.do?page=A02060400 $\& d c=K 02060400 \&$ boardId $=1030 \& c p=13$ (Korean).

2. Pramis J. Number of mobile phones to exceed world population by 2014; 2013 [cited 2016 Jun 27]. Available from: http://www.digitaltrends.com/mobile/mobile-phone-world-population-2014/.

3. Meo SA, Alsubaie Y, Almubarak Z, Almutawa H, AlQasem Y, Hasanato RM. Association of exposure to radio-frequency electromagnetic field radiation (RF-EMFR) generated by mobile phone base stations with glycated hemoglobin ( $\mathrm{HbA1c}$ ) and risk of type 2 diabetes mellitus. Int J Environ Res Public Health 2015;12(11):14519-14528.

4. Röösli M, Hug K. Wireless communication fields and non-specific symptoms of ill health: a literature review. Wien Med Wochenschr 2011;161(9-10):240-250.

5. Hardell L, Carlberg M, Hansson Mild K. Case-control study on cellular and cordless telephones and the risk for acoustic neuroma or meningioma in patients diagnosed 2000-2003. Neuroepidemiology 2005;25(3):120-128.

6. Hardell L, Carlberg M, Mild KH. Case-control study of the association between the use of cellular and cordless telephones and malignant brain tumors diagnosed during 2000-2003. Environ Res 2006;100(2):232-241.

7. International Agency for Research on Cancer. Non-ionizing radiation, part 2: radiofrequency electromagnetic fields [cited 2016 Jun 27]. Available from: http://monographs.iarc.fr/ENG/Monographs/ vol102/mono102.pdf.

8. Yoon S, Choi JW, Lee E, An H, Choi HD, Kim N. Mobile phone use and risk of glioma: a case-control study in Korea for 20022007. Environ Health Toxicol 2015;30:e2015015.

9. Repacholi MH, Lerchl A, Röösli M, Sienkiewicz Z, Auvinen A, Breckenkamp J, et al. Systematic review of wireless phone use and brain cancer and other head tumors. Bioelectromagnetics 2012; 33(3):187-206. 
10. Röösli M. Radiofrequency electromagnetic field exposure and non-specific symptoms of ill health: a systematic review. Environ Res 2008;107(2):277-287.

11. Yzermans J, Baliatsas C, van Dulmen S, Van Kamp I. Assessing nonspecific symptoms in epidemiological studies: development and validation of the symptoms and perceptions $(\mathrm{SaP})$ questionnaire. Int J Hyg Environ Health 2016;219(1):53-65.

12. Szyjkowska A, Gadzicka E, Szymczak W, Bortkiewicz A. The risk of subjective symptoms in mobile phone users in Poland--an epidemiological study. Int J Occup Med Environ Health 2014;27(2):293303.

13. Sudan M, Kheifets L, Arah O, Olsen J, Zeltzer L. Prenatal and postnatal cell phone exposures and headaches in children. Open Pediatr Med Journal 2012;6(2012):46-52.

14. Mortazavi SM, Ahmadi J, Shariati M. Prevalence of subjective poor health symptoms associated with exposure to electromagnetic fields among university students. Bioelectromagnetics 2007;28(4):326330.

15. Kim H, Yun CH, Thomas RJ, Lee SH, Seo HS, Cho ER, et al. Obstructive sleep apnea as a risk factor for cerebral white matter change in a middle-aged and older general population. Sleep 2013;36(5):709$715 B$.

16. Bayliss MS, Dewey JE, Dunlap I, Batenhorst AS, Cady R, Diamond $\mathrm{ML}$, et al. A study of the feasibility of Internet administration of a computerized health survey: the headache impact test (HIT). Qual Life Res 2003;12(8):953-961.

17. Chu MK, Im HJ, Ju YS, Yu KH, Ma HI, Kim YJ, et al. Validity and reliability assessment of Korean Headache Impact Test-6 (HIT-6). J Korean Neurol Assoc 2009;27(1):1-6 (Korean).

18. Jang S. Collection of health statistics and standardization of measurement. Seoul: Gyechuk Munwhasa; 2000, p. 92-132 (Korean).

19. Lee SK, Yoon DW, Yi H, Lee SW, Kim JY, Kim JK, et al. So-eum type as an independent risk factor for irritable bowel syndrome: a population-based study in Korea.J Altern Complement Med 2014; 20(11):846-852.

20. Beck AT, Ward CH, Mendelson M, Mock J, Erbaugh J. An inventory for measuring depression. Arch Gen Psychiatry 1961;4:561-571.

21. Won CW, Rho YG, SunWoo D, Lee YS. The validity and reliability of Korean Instrumental Activities of Daily Living (K-IADL) scale.J Korean Geriatr Soc 2002;6(4):273-280 (Korean).

22. Lee J, Shin C, Ko YH, Lim J, Joe SH, Kim S, et al. The reliability and validity studies of the Korean version of the Perceived Stress Scale. Korean J Psychosom Med 2012;20(2):127-134 (Korean).

23. Cohen S, Kamarck T, Mermelstein R. A global measure of perceived stress. J Health Soc Behav 1983;24(4):385-396.

24. Buysse DJ, Reynolds CF 3rd, Monk TH, Berman SR, Kupfer DJ. The Pittsburgh Sleep Quality Index: a new instrument for psychiatric practice and research. Psychiatry Res 1989;28(2):193-213.

25. Sohn SI, Kim DH, Lee MY, Cho YW. The reliability and validity of the Korean version of the Pittsburgh Sleep Quality Index. Sleep Breath 2012;16(3):803-812.
26. Ware J Jr, Kosinski M, Keller SD. A 12-Item Short-Form Health Survey: construction of scales and preliminary tests of reliability and validity. Med Care 1996;34(3):220-233.

27. Chae H, Kim YH, Park SH, Hwang EH, Kwon YK, Lee SJ. Study on the effects of Sasang psychobiological factors on general health status. Korean J Orient Physiol Pathol 2014;28(4):452-459 (Korean).

28. Nachit-Ouinekh F, Dartigues JF, Henry P, Becg JP, Chastan G, Lemaire $\mathrm{N}$, et al. Use of the headache impact test (HIT-6) in general practice: relationship with quality of life and severity. Eur J Neurol 2005;12(3):189-193.

29. K Sri N. Mobile phone radiation: physiological \& pathophysiologcal considerations. Indian J Physiol Pharmacol 2015;59(2):125135.

30. Zhu Y, Jin W, Liu H, Peng D, Ding Z, Tang Z, et al. Effects of electromagnetic fields from mobile phones on depression and anxiety after titanium mesh cranioplasty among patients with traumatic brain injury. Brain Inj 2016;30(1):66-73.

31. Coureau G, Bouvier G, Lebailly P, Fabbro-Peray P, Gruber A, Leffondre $\mathrm{K}$, et al. Mobile phone use and brain tumours in the CERENAT case-control study. Occup Environ Med 2014;71(7):514522.

32. INTERPHONE Study Group. Brain tumour risk in relation to mobile telephone use: results of the INTERPHONE international case-control study. Int J Epidemiol 2010;39(3):675-694.

33. Berg-Beckhoff G, Blettner M, Kowall B, Breckenkamp J, Schlehofer B, Schmiedel S, et al. Mobile phone base stations and adverse health effects: phase 2 of a cross-sectional study with measured radio frequency electromagnetic fields. Occup Environ Med 2009; 66(2):124-130.

34. Söderqvist F, Carlberg M, Hardell L. Use of wireless telephones and self-reported health symptoms: a population-based study among Swedish adolescents aged 15-19 years. Environ Health 2008; 7:18.

35. Zheng F, Gao P, He M, Li M, Tan J, Chen D, et al. Association between mobile phone use and self-reported well-being in children: a questionnaire-based cross-sectional study in Chongqing, China. BMJ Open 2015;5(5):e007302.

36. Khan MM. Adverse effects of excessive mobile phone use. Int J Occup Med Environ Health 2008;21(4):289-293.

37. Sandström M, Wilen J, Oftedal G, Hansson Mild K. Mobile phone use and subjective symptoms. Comparison of symptoms experienced by users of analogue and digital mobile phones. Occup Med (Lond) 2001;51(1):25-35.

38. Chu MK, Song HG, Kim C, Lee BC. Clinical features of headache associated with mobile phone use: a cross-sectional study in university students. BMC Neurol 2011;11:115.

39. Shum M, Kelsh MA, Sheppard AR, Zhao K. An evaluation of selfreported mobile phone use compared to billing records among a group of engineers and scientists. Bioelectromagnetics 2011;32(1): $37-48$. 